\title{
Shrinkage of excluded aneurysmal spaces: The excellent results of endovascular stent grafting through the aortic arch for distal arch aneurysm
}

Masanobu Watari, MD, Taijiro Sueda, MD, Osamu Ishii, MD, Katsuhiko Imai, MD, Shibamura Hidenori, MD, Hiroshi Sakai, MD, Hideichi Wada, MD, Kenji Okada, MD, and Kazumasa Orihashi, MD, Hiroshima, Japan

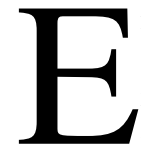
ndoluminal repair is currently the treatment of choice for aortic arch aneurysm. Transfemoral stent grafting is less invasive, but we believe that it is not reliable, that it is difficult, and that its indications and prognosis need more discussion. On the other hand, open stent grafting, endovascular stent grafting through the aortic arch, is more invasive but is a reliable and easy technique. Therefore, we prefer the latter

From the Department of Surgery, Hiroshima University School of Medicine, Hiroshima, Japan.

Received for publication Dec 28, 2000; accepted for publication Feb 20, 2001.

Address for reprints: Masanobu Watari, MD, First Department of Surgery, Hiroshima University School of Medicine, Kasumi 1-2-3, Minami-ku,

Hiroshima, 734-8551, Japan (E-mail: mackie@mcai.med.hiroshima-u.ac.jp).

J Thorac Cardiovasc Surg 2001;122:829-32

Copyright @ 2001 by The American Association for Thoracic Surgery

$0022-5223 / 2001 \$ 35.00+0 \quad \mathbf{1 2 / 5 4 / 1 1 5 2 3 4}$

doi:10.1067/mtc.2001.115234 method. We started performing open stent grafting in 1997. By now, our open stent-grafting technique has now progressed to a safe and reliable method by adding a few revisions and modifications. ${ }^{1-3}$

In this report we discuss the postoperative changes in the excluded aneurysmal space of the distal aortic arch aneurysm in patients we treated by means of open stent grafting because we believe it is important to determine whether the excluded aneurysmal space shrinks.

\section{Clinical Summary}

Procedures were as follows:

- A median sternal approach is adopted.

- Normograde extracorporeal circulation through the ascending aorta or the right axillary artery is essential.

- Concomitant cardiac repair is performed while decreasing the body temperature.

- Hypothermic circulatory arrest and selective cerebral perfusion are instituted at a rectal temperature of $25^{\circ} \mathrm{C}$. The stent graft is delivered through a small incision on the aortic arch below the left subclavian artery. 


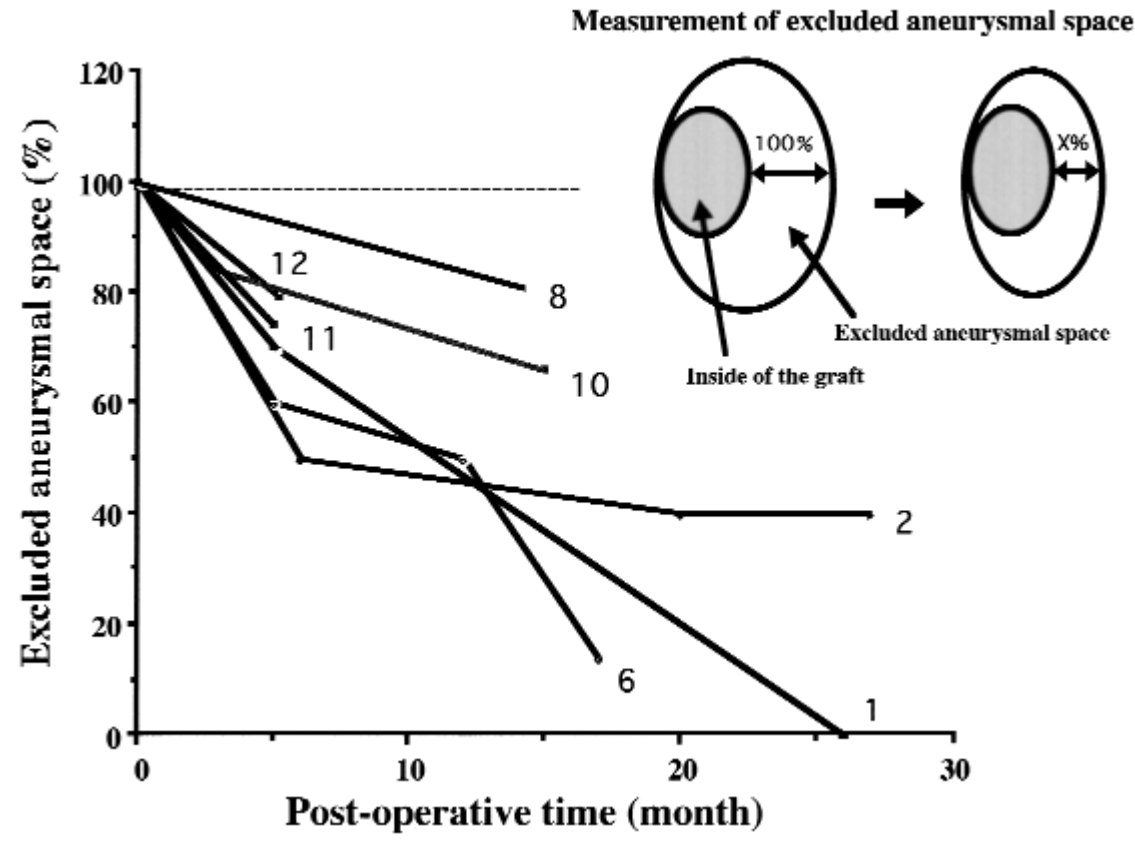

Figure 1. Individual changes in the excluded aneurysmal space are presented.

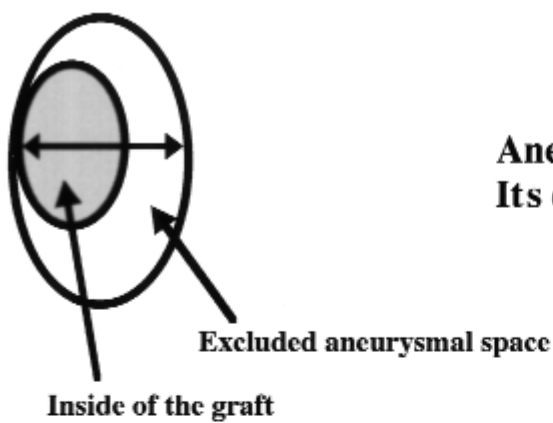

Aneurysmal diameter is measured. Its direction is vertical to graft axis.

Inside of the graft

(cm)

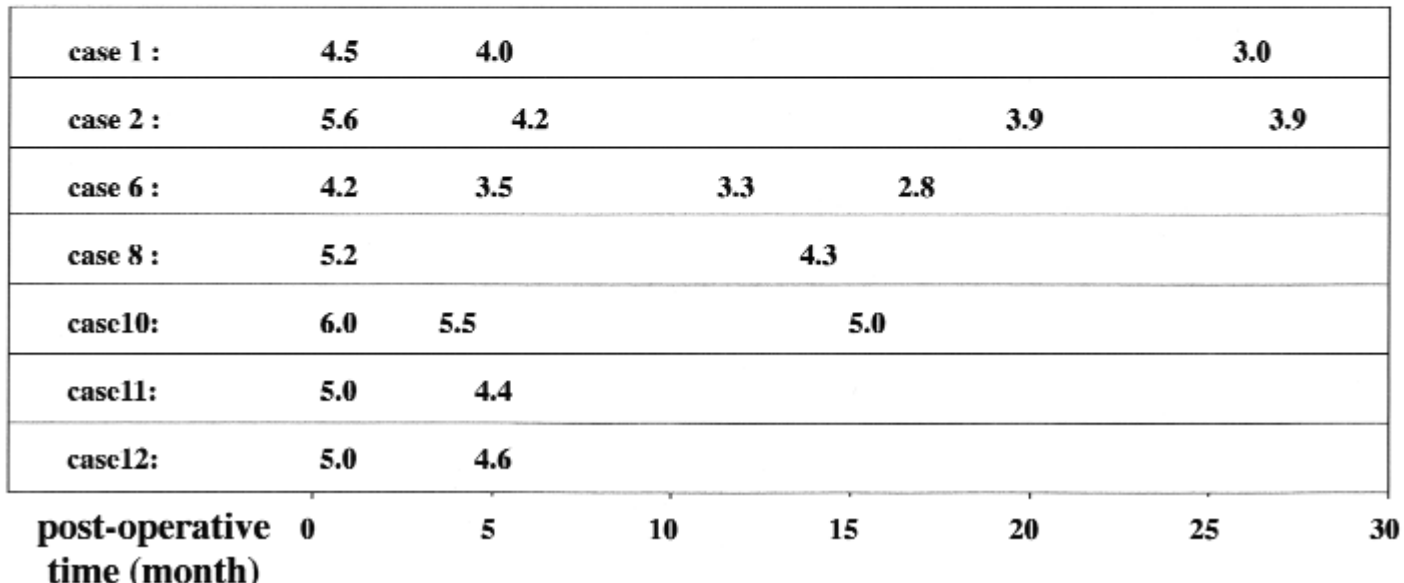

Figure 2. Change of aneurysmal diameter.

- A proximal anastomosis of the graft and the aortic arch, including the small incision, is carefully made.

- Transesophageal echocardiography is used $^{3}$ to evaluate the condition of the aneurysm and the aortic wall, to determine the graft size by measuring the descending aortic diameter, to monitor and guide the stent-graft delivery, to tailor the distal tip of the stent graft, and to confirm the absence of endoleak into the excluded aneurysmal space. 


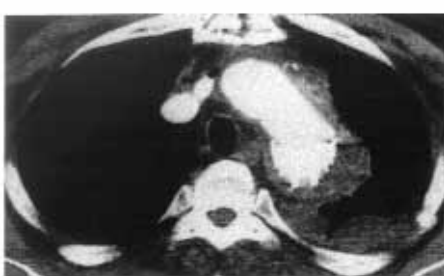

Two weeks after surgery

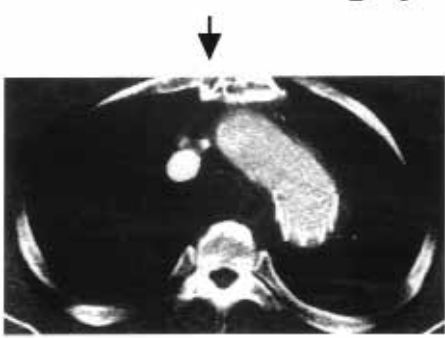

Twenty six months after surgery The aneurysmal space has vanished.

Case 1 (Male, 64y)

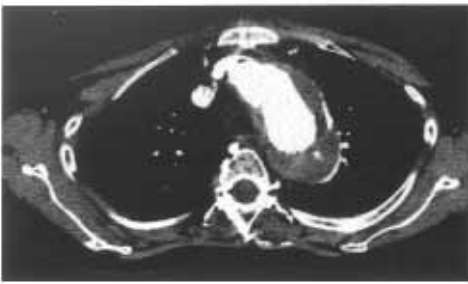

Two weeks after surgery

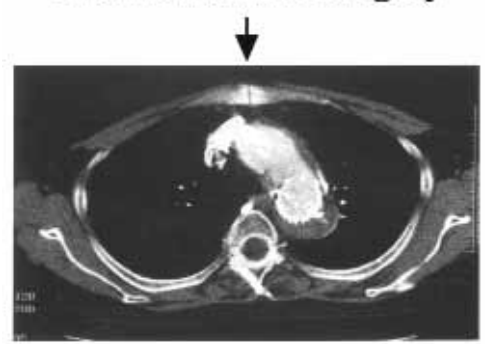

Five months after surgery The aneurysmal space shrunk.

Case 6 (Female, 65y)

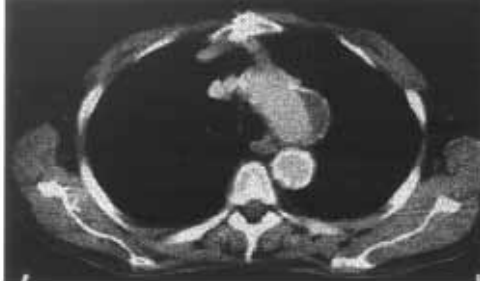

Two weeks after surgery

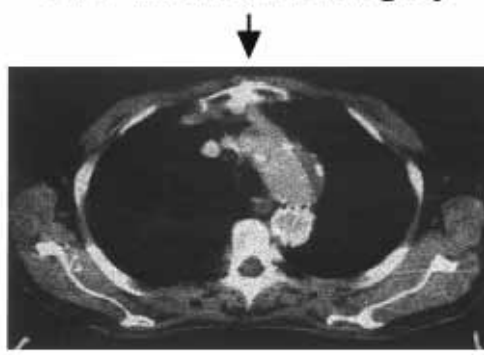

Five months after surgery

Case 11 (Male, 70y)

Figure 3. CT findings in typical patients.

Device. The stent graft used consisted of a Gianturco Z stent with a diameter of $45 \mathrm{~mm}$ (Cook Inc, Bloomington, Ind) and a Dacron graft (porosity of $250 \mathrm{~mL} / \mathrm{m}^{2}$; Intervascular Inc, Clearwater, Fla). The stent is fixed inside the graft by means of monofilament sutures. Various sizes of stent grafts were prepared and sterilized in advance. After measuring the distance from intima to intima of the descending aorta by means of transesophageal echocardiography, we chose a stent graft one size bigger than the aortic diameter. When the stent graft was delivered, the tip of the stent graft was monitored by means of transesophageal echocardiography. If the tip was not fully dilated, a balloon catheter was used to expand the tip into the aorta.

Indications. In cases of distal aortic arch aneurysm, the condition of the descending aorta determines whether it can tolerate the stent graft, and the stent-graft tip must be placed far from the diaphragm $(\geq 9 \mathrm{~cm})$.

Fourteen patients with distal aortic arch aneurysms were treated from September 1997 to August 2000. Twelve patients are currently alive, but 2 have died. Endoleak was found in only 1 patient, who died of pneumonia 6 months postoperatively. A follow-up computed tomography (CT) scan was performed for the 7 living patients. In these 7 patients the excluded aneurysmal space size was followed by comparing it with the initial size, which was confirmed within 1 month after the operation. The maximum dimension of the excluded aneurysmal space was measured and compared at the same level of the CT slice. The maximum aneurysmal diameter was also measured at the same level of the CT slice.

The serial changes in these 7 patients are shown in Figures 1 and 2. It is clear that the excluded aneurysmal space shrunk or vanished in all patients. Three examples of CT findings are shown in Figure 3.

\section{Discussion}

Endovascular stent grafting through the aortic arch for distal arch aneurysm has the following merits: a distal anastomosis is unnecessary, and there is a decrease in the bleeding and operation time, which leads to a less invasive operation compared with conventional surgical repair. Endovascular stent grafting through the aortic arch for a distal arch aneurysm was first reported by Kato and colleagues. ${ }^{4}$ Our procedure is basically similar to their method, but it has been modified in area to avoid complications, such as cerebral infarction, paraplegia, and endoleak. ${ }^{1-3}$ In short, a normograde extracorporeal circulation is adopted in principle, and transesophageal echocardiography is used in many ways.

The purpose of endoluminal stent grafting is to isolate the aneurysm from the blood stream. It is expected that the aneurysmal space will shrink after rapid thrombus formation if the aneurysm is perfectly excluded. In this sense the shrinkage of the excluded aneurysm translates into a success for the treatment. Therefore, considering the fact that all 7 patients examined with follow-up CT scans showed excluded aneurysmal space shrinkage, we conclude that open stent grafting is a satisfactory method and one that has a good prognosis.

Transfemoral stent grafting is less invasive and has great potential to become a less invasive therapeutic modality as opposed to conventional surgical therapies. However, its performance is unreliable at present because of the high incidence of endoleaks. ${ }^{5}$ Therefore, we must wait for the development of improved devices and techniques for this approach. On the other hand, although endovascular stent grafting through the aortic arch seems rather invasive, it is a more reliable and easier option for the surgeon. On the basis of our experience, we strongly recommend this option. 


\section{References}

1. Sueda T, Watari M, Orihashi K, Shikata H, Matsuura Y. Endovascular stent-grafting via the aortic arch for distal arch aneurysm: an alternative of endovascular stent-grafting in a complicated case. Ann Thorac Cardiovasc Surg. 1999;5:206-8.

2. Sueda T, Watari M, Okada K, Orihashi K, Matsuura Y. Endovascular stent-grafting through the aortic arch: an alternative approach for distal arch aortic aneurysm. Ann Thorac Surg. 2000;70:1251-4.

3. Orihashi K, Matsuura Y, Sueda T, Watari M, Okada K, Sugawara Y, et al. Echocardiography-assisted surgery in transaortic endovascular stent grafting: role of transesophageal echocardiography. $J$ Thorac Cardiovasc Surg. 2000;120:672-8.

4. Kato M, Ohnishi K, Kaneko M, Ueda T, Kishi D, Mizushima T, et al. New graft-implanting method for thoracic aortic aneurysm or dissection with a stented graft. Circulation. 1996;94(Suppl):II-188-93.

5. Kawaguchi S, Ishimaru S, Shimazaki T, Yokoi Y, Koizumi N, Obitsu $\mathrm{Y}$, et al. Clinical results of endovascular stent graft repair for fifty cases of thoracic aortic aneurysms. Jpn J Thorac Cardiovasc Surg. 1998;46:971-5 